\title{
Reverse Poincaré-type inequalities for the difference of superharmonic functions
}

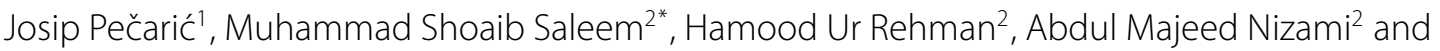
Abid Hussain²

\section{"Correspondence:}

shaby455@yahoo.com

${ }^{2}$ Department of Mathematics,

University of Education, Lahore,

Pakistan

Full list of author information is

available at the end of the article

\begin{abstract}
In this paper, we develop the weighted square integral inequalities for the difference of two smooth superharmonic functions. Then we prove the existence and integrability of the Sobolev derivative for superharmonic functions. The inequalities are generalized for the difference of two weak superharmonic functions. We also establish that the superharmonic approximation is indeed the better imitation of the exact unknown solution rather than the usual uniform approximation.
\end{abstract}

Keywords: concave functions; superharmonic functions; weak derivative; weight function; compact support; weak superharmonic function; superharmonic majorants

\section{Introduction and statement of the main result}

The role of mathematical inequalities within the mathematical branches as well as in its enormous applications should not be underestimated. The square integral estimate for the first derivative of convex function was established in $[1,2]$. Then the results were improved by Hussain et al. in [3]. Such kinds of inequalities are very useful for the hedging problems in mathematical finance.

The negative of a convex function is concave (concave down) functions. It is well known in modern calculus that the natural generalization of concave functions to a function of several independent variables is a superharmonic function, related to the famous Laplace operator. So it is also interesting to develop similar inequalities for the superharmonic functions. The latter functions are often considered as a powerful tool for the study of solvability of the classical Poisson and Dirichlet problems in the theory of partial differential equations.

Throughout the paper we will use the following notations:

$D, D \subset R^{n}$ is bounded and having a smooth boundary, $B=B\left(x_{0}, r\right)$ is the open ball in $R^{n}$ with center $x_{0}$ and radius $r(r>0), \bar{B}$ is its closure. $L^{\infty}(B)$ is the space of bounded (a.e. $d x$ ) on $B$.

$\Delta$ is the $n$ dimensional Laplace operator.

$C_{0}^{2}(B)$ is the space of twice continuously differentiable functions having compact support on $B$.

$u(x)$ is said to be smooth superharmonic if $u(x) \in C^{2}(B)$,

$$
\Delta u(x) \leq 0
$$

(c) 2015 Pečarić et al. This article is distributed under the terms of the Creative Commons Attribution 4.0 International License (http://creativecommons.org/licenses/by/4.0/), which permits unrestricted use, distribution, and reproduction in any medium, provided you give appropriate credit to the original author(s) and the source, provide a link to the Creative Commons license, and indicate if changes were made. 
A bounded measurable function $u(x)$ defined on the ball $B$ is said to be weak superharmonic if for all non-negative functions $\phi \in C_{0}^{2}(B)$ the following holds:

$$
\int_{B} u(x) \Delta \phi(x) d x \leq 0
$$

We will consider the arbitrary smooth weight function satisfying the following:

$$
\begin{aligned}
& h(x) \geq 0 \quad \text { if } x \in D, \\
& h(x)=\frac{\partial h(x)}{\partial x_{i}}=0, \quad i=1,2, \ldots, n, x \in \partial D .
\end{aligned}
$$

We will also take the particular form of the weight function for the ball $B\left(x_{0}, r\right)$ in the following way:

$$
h(x)=\left(r^{2}-\left|x-x_{0}\right|^{2}\right)^{2} .
$$

We will find

$$
\frac{\partial h(x)}{\partial x_{i}}=4\left(x_{i}-x_{i}^{0}\right)\left(r^{2}-\left|x-x_{0}\right|^{2}\right), \quad i=1,2, \ldots, n .
$$

It is clear by the definition of the weight function that $h(x)=\frac{\partial h(x)}{\partial x_{i}}=0, i=1,2, \ldots, n$, for $x$ on the boundary of ball $B\left(x_{0}, r\right)$.

Now we formulate our main result.

Theorem 1.1 Consider two arbitrary continuous weak superharmonic functions $u_{i}(x), i=$ 1,2 , on the ball $B, B=B\left(x_{0}, r\right)$, then the following energy estimate holds:

$$
\begin{aligned}
& \int_{B}\left|\operatorname{grad} u_{2}(x)-\operatorname{grad} u_{1}(x)\right|^{2} h(x) d x \\
& \quad \leq \frac{1}{2}\left\|u_{2}-u_{1}\right\|_{L_{\infty}(B)}^{2}+\left(\left\|u_{2}-u_{1}\right\|_{L_{\infty}(B)}\right)\left(\left\|u_{1}\right\|_{L_{\infty}(B)}+\left\|u_{2}\right\|_{L_{\infty}(B)}\right) \int_{B}|\Delta h(x)| d x,
\end{aligned}
$$

where $h(x)$ is the weight function defined in (1.5).

We will organize the paper in the following way: In the second section we will establish the inequality for the smooth superharmonic functions and then by a standard mollification technique we will approximate the weak superharmonic functions by the smooth ones. In the last section we will prove the existence and integrability of weak superharmonic functions and then establish the proof of our main result. At the end we will also explain that a superharmonic approximation is better than the usual uniform approximation.

\section{The case of smooth superharmonic functions and mollification of weak superharmonic functions}

Our starting point will be the following theorem.

Theorem 2.1 Consider two arbitrary smooth superharmonic functions $u_{i}(x), i=1,2$ over the domain $D, D \subset R^{n}$ (this domain is bounded, having a smooth boundary), i.e. $u_{i}(x) \in$ 
$\overline{C^{2}} D, i=1,2$, and $\Delta u_{i}(x) \leq 0$ if $x \in D, i=1,2$. Then the following holds:

$$
\int_{D}\left|\operatorname{grad} u_{2}(x)-\operatorname{grad} u_{1}(x)\right|^{2} h(x) d x \leq\|\tilde{u}(x)\|_{L_{p}(D)}\|\Delta h(x)\|_{L_{q}(D)},
$$

where $p$ and $q$ are conjugates and

$$
\tilde{u}(x)=\frac{1}{2}\left(u_{2}(x)-u_{1}(x)\right)^{2}-\left\|u_{2}(x)-u_{1}(x)\right\|_{L_{\infty}}\left(u_{2}(x)+u_{1}(x)\right)
$$

and $h(x)$ is the weight function defined in (1.3).

Proof Let us denote

$$
u(x)=u_{2}(x)-u_{1}(x)
$$

Take

$$
\begin{aligned}
& \int_{D}|\operatorname{grad} u(x)|^{2} h(x) d x \\
& \quad=\int_{D}\left(\left(\frac{\partial u}{\partial x_{1}}\right)^{2}+\left(\frac{\partial u}{\partial x_{2}}\right)^{2}+\cdots+\left(\frac{\partial u}{\partial x_{n}}\right)^{2}\right) h(x) d x \\
& =\int_{D}\left(\frac{\partial u}{\partial x_{1}}\right)^{2} h(x) d x+\int_{D}\left(\frac{\partial u}{\partial x_{2}}\right)^{2} h(x) d x+\cdots+\int_{D}\left(\frac{\partial u}{\partial x_{n}}\right)^{2} h(x) d x
\end{aligned}
$$

Take the first integral on the right hand side,

$$
\int_{D}\left(\frac{\partial u}{\partial x_{1}}\right)^{2} h(x) d x=\int_{D} \frac{\partial u}{\partial x_{1}}\left(\frac{\partial u}{\partial x_{1}} h(x)\right) d x
$$

Using integration by parts and the fact that the weight function vanishes on the boundary of the domain, we get

$$
\begin{aligned}
& =-\int_{D} u(x) \frac{\partial^{2} u}{\partial x_{1}^{2}} h(x) d x-\int_{D} u(x) \frac{\partial u}{\partial x_{1}} \frac{\partial h(x)}{\partial x_{1}} \\
& =-\int_{D} u(x) \frac{\partial^{2} u}{\partial x_{1}^{2}} h(x) d x-\frac{1}{2} \int_{D} \frac{\partial u^{2}(x)}{\partial x_{1}} \frac{\partial h(x)}{\partial x_{1}} d x .
\end{aligned}
$$

Again using integration by parts on the second integral and also the definition of the weight function, we obtain

$$
=-\int_{D} u(x) \frac{\partial^{2} u}{\partial x_{1}^{2}} h(x) d x+\frac{1}{2} \int_{D} u^{2}(x) \frac{\partial^{2} h(x)}{\partial x_{1}^{2}} d x .
$$

Solving all integrals in a similar way, (2.3) becomes

$$
\begin{aligned}
\int_{D}|\operatorname{grad} u(x)|^{2} h(x) d x & =\frac{1}{2} \int_{D} u^{2}(x) \Delta h(x) d x-\int_{D} u(x) \Delta u(x) h(x) d x \\
& \leq \frac{1}{2} \int_{D} u^{2}(x) \Delta h(x) d x+\sup _{x \in D}|u(x)| \int_{D}|\Delta u(x)| h(x) d x
\end{aligned}
$$

From (2.2) we have $|\Delta u(x)| \leq\left|\Delta u_{2}(x)\right|+\left|\Delta u_{1}(x)\right|$. 
Since $u_{1}$ and $u_{2}$ are subharmonic, we have

$$
\begin{aligned}
& \left|\Delta u_{2}(x)\right|=-\Delta u_{2}(x), \\
& \left|\Delta u_{1}(x)\right|=-\Delta u_{1}(x) .
\end{aligned}
$$

So the above becomes

$$
\begin{aligned}
\int_{D}|\operatorname{grad} u(x)|^{2} h(x) d x \leq & \frac{1}{2} \int_{D} u^{2}(x) \Delta h(x) d x \\
& -\sup |u(x)| \int_{D}\left(\Delta\left(u_{2}(x)+u_{1}(x)\right)\right) h(x) d x .
\end{aligned}
$$

Using the Green theorem and the fact that $h(x)$ and its derivative vanish on the boundary of the domain, we have

$$
\begin{aligned}
& \int_{D}|\operatorname{grad} u(x)|^{2} h(x) d x \\
& \leq \frac{1}{2} \int_{D} u^{2}(x) \Delta h(x) d x-\sup _{x \in D}|u(x)| \int_{D}\left(u_{2}(x)+u_{1}(x)\right) \Delta h(x) d x \\
& \quad \leq \int_{D}\left[\frac{u^{2}(x)}{2}-\|u(x)\|_{L^{\infty}}\left(u_{2}(x)+u_{1}(x)\right)\right] \Delta h(x) d x \\
& \int_{D}|\operatorname{grad} u(x)|^{2} h(x) d x \leq \int_{D}\left|\frac{u^{2}(x)}{2}-\|u(x)\|_{L^{\infty}}\left(u_{2}(x)+u_{1}(x)\right) \Delta h(x)\right| d x .
\end{aligned}
$$

Finally using the Hölder inequality yields the required result.

Remark 2.2 Using the definition of modulus on (2.5) we obtain the following inequality:

$$
\begin{aligned}
\int_{D}|\operatorname{grad} u(x)|^{2} h(x) d x \leq & \frac{1}{2}\left\|u_{2}(x)-u_{1}(x)\right\|_{L^{\infty}(D)}^{2}+\left\|u_{2}(x)-u_{1}(x)\right\|_{L^{\infty}(D)} \\
& \times\left(\left\|u_{2}(x)\right\|_{L^{\infty}(D)}+\left\|u_{1}(x)\right\|_{L^{\infty}(D)}\right) \int_{D}|\Delta h(x)| d x
\end{aligned}
$$

Writing the above remark for an arbitrary ball $B, B=B\left(x_{0}, r\right) \subset R^{n}$, we get

\section{Remark 2.3}

$$
\begin{aligned}
\int_{B}|\operatorname{grad} u(x)|^{2} h(x) d x \leq & \frac{1}{2}\left\|u_{2}(x)-u_{1}(x)\right\|_{L^{\infty}(B)}^{2}+\left\|u_{2}(x)-u_{1}(x)\right\|_{L^{\infty}(B)} \\
& \times\left(\left\|u_{2}(x)\right\|_{L^{\infty}(B)}+\left\|u_{1}(x)\right\|_{L^{\infty}(B)}\right) \int_{B}|\Delta h(x)| d x .
\end{aligned}
$$

Now we approximate the weak superharmonic function (1.2) by the smooth ones. To this aim, we will use the classical mollification technique.

Define

$$
\eta(x)= \begin{cases}c \exp \frac{1}{x^{2}-1} & \text { if }|x|<1 \\ 0 & \text { if }|x| \geq 1\end{cases}
$$

where $x \in R^{n}, c>0$ is constant such that $\int_{R^{n}} \eta(x) d x=1$. 
Let us define the mollification of the bounded measurable function $u(x)$ on the ball $B$

$$
u_{\epsilon}(x)=\int_{B} \eta\left(\frac{x-y}{\epsilon}\right) u(y) d y
$$

Let us denote $\eta_{\epsilon}(x-y)=\eta\left(\frac{x-y}{\epsilon}\right)$. Then it is clear that

$$
\frac{\partial^{2}}{\partial x_{i}^{2}} \eta_{\epsilon}(x-y)=\frac{\partial^{2}}{\partial y_{i}^{2}} \eta_{\epsilon}(x-y), \quad \forall i=1,2, \ldots, n
$$

Using (2.10) in (2.9), we have

$$
\Delta_{x} u_{\epsilon}(x)=\int_{B} u(y) \cdot \Delta_{y} \eta_{\epsilon}(x-y) d y
$$

where $\Delta_{x}$ and $\Delta_{y}$ are the Laplace operator with respect to $\mathrm{x}$ and $\mathrm{y}$, respectively. Also define the balls $B_{k}$ in the following way:

$$
B_{k}=B\left(x_{0}, r_{k}\right) \quad \text { where } r_{k}=r\left(\frac{k+1}{k+2}\right) \text {. }
$$

The following theorem states that the functions $u_{\epsilon}(x)$ are smooth superharmonic functions on $B_{k}$ for sufficiently small $\epsilon$.

Theorem 2.4 Let $u(x)$ be a weak superharmonic function on the ball $B=B\left(x_{0}, r\right)$. Then for any $k=1,2,3, \ldots$ there exists $\hat{\epsilon}>0$ such that for $0<\epsilon<\hat{\epsilon}$ each function $u_{\epsilon}(x)$ is smooth superharmonic on the ball $B_{k}$, that is,

$$
\Delta u_{\epsilon}(x) \leq 0 \quad \text { if } x \in B_{k}
$$

Proof Take $\hat{\epsilon}=\frac{r}{2(k+2)}$. By definition it is trivial that $u_{\epsilon}(x), \epsilon>0$, is infinitely differentiable w.r.t. $x$. Now we will see that for arbitrary $x \in B_{k}$ the function $\eta_{\epsilon}(x-y)$ has compact support on $B$ as a function of $y$.

Take the ball $\hat{B_{k}}$ in the following way:

$$
\hat{B_{k}}=B\left(x_{0}, \frac{2 k+3}{2 k+4} r\right) \text {. }
$$

Take $y \in \hat{B}_{k}$, then

$$
|y-x|>\frac{1}{2(k+2)} r>\epsilon .
$$

Hence we have $\eta_{\epsilon}(x-y)=0$. Therefore the non-negative function $\eta_{\epsilon}(x-y)$ has a compact support in $B$ as a function of $y$. So by the definition of a weak superharmonic function $u(x)$, we have

$$
\int_{B} u(y) \cdot \Delta \eta_{\epsilon}(x-y) \leq 0 .
$$

From (2.10) we get $\Delta u_{\epsilon}(x) \leq 0$ if $x \in B_{k}$ and $\epsilon<\hat{\epsilon}$. 


\section{Sobolev gradient existence and proof of the main result}

Let us introduce the weight function $h_{k}(x)$ corresponding to the balls $B_{k}$

$$
h_{k}(x)=\left(r_{k}^{2}-\left|x-x_{0}\right|^{2}\right)^{2}, \quad x \in \bar{B}, k=1,2, \ldots
$$

The following theorem will show the existence of a weak derivative and square integrability with respect to the weight function.

Theorem 3.1 Let $u(x)$ be a continuous weak superharmonic function, then it has weak partial derivatives $\frac{\partial u(x)}{\partial x_{i}}, i=1,2, \ldots, n$, in the ball $B$ and they are square integrable with respect to the weight function $h(x)$, i.e.

$$
\int_{B\left(x_{0}, r\right)}|\operatorname{grad} u(x)|^{2} h(x) d x<\infty
$$

Proof If $u(x)$ is continuous in the ball $B$ then on any compact set $K, K \subset B$, we have the uniform convergence (see, for example, the work by Evans),

$$
\sup _{K}\left|u_{\epsilon}(x)-u(x)\right| \rightarrow 0, \quad \epsilon \rightarrow 0,
$$

where $u_{\epsilon}(x)$ is the mollification of the weak superharmonic function $u(x)$.

Taking $\epsilon=\frac{1}{m}, m=1,2, \ldots$, the latter convergence takes the form

$$
\sup _{K}\left|u_{m}(x)-u(x)\right| \rightarrow 0, \quad m \rightarrow \infty .
$$

Since by definition it is clear that $B_{k} \subset \subset B$ (compactly embedded) we see from Remark 2.3 for any $k$ that there is such an $m_{k}$ that each $u_{m}(x)$ is smooth subharmonic in the ball $B_{k}$ if $m \geq m_{k}$.

Now writing the inequality (2.6) for the ball $B_{k+l}$ and for the functions

$$
u_{1}(x)=u_{m}(x), \quad u_{2}(x)=u_{p}(x), \quad m, p \geq m_{k+l}
$$

we get

$$
\begin{aligned}
& \int_{B_{k+l}}\left|\operatorname{grad} u_{p}(x)-\operatorname{grad} u_{m}(x)\right|^{2} h_{k+l}(x) d x \\
& \quad \leq \frac{1}{2}\left\|u_{p}(x)-u_{m}(x)\right\|_{L^{\infty} B_{k+l}}^{2}+\left\|u_{p}(x)-u_{m}(x)\right\|_{L^{\infty}\left(B_{k+l}\right)}\left(\left\|u_{p}(x)\right\|_{L^{\infty}\left(B_{k+l}\right)}\right. \\
& \left.\quad+\left\|u_{m}(x)\right\|_{L^{\infty}\left(B_{k+l}\right)}\right) \int_{B_{k+l}}|\Delta h(x)| d x .
\end{aligned}
$$

Let us denote

$$
\alpha_{k+l}=\int_{B_{k+l}}\left|\Delta h_{k+l}\right| d x
$$

and

$$
\widehat{\alpha}=\inf _{x \in B_{k}} h_{k+l}(x) .
$$


Also by the definition $B_{k} \subset B_{k+l}$, so the above can be written as

$$
\begin{aligned}
& \widehat{\alpha} \int_{B_{k}}\left|\operatorname{grad} u_{p}(x)-\operatorname{grad} u_{m}(x)\right|^{2} d x \\
& \quad \leq \alpha_{k+l}\left[\frac{1}{2}\left\|u_{p}(x)-u_{m}(x)\right\|_{L^{\infty} B_{k+l}}^{2}+\left\|u_{p}(x)-u_{m}(x)\right\|_{L^{\infty}\left(B_{k+l}\right)}\left(\left\|u_{p}(x)\right\|_{L^{\infty}\left(B_{k+l}\right)}\right.\right. \\
& \left.\left.\quad+\left\|u_{m}(x)\right\|_{L^{\infty}\left(B_{k+l}\right)}\right)\right]
\end{aligned}
$$

We also have

$$
\left\|u_{p}(x)-u_{m}(x)\right\| \rightarrow 0, \quad \text { if } m, p \rightarrow \infty
$$

Taking the limit $m, p \rightarrow \infty$ on (3.6), we get

$$
\lim _{m, p \rightarrow \infty} \sum_{i=1}^{n} \int_{B_{k}}\left(\frac{\partial u_{p}(x)}{\partial x_{i}}-\frac{\partial u_{m}(x)}{\partial x_{i}}\right)^{2} d x=0
$$

Since $L^{2}\left(B_{k}\right)$ is complete, $\exists$ a family of measurable function $v_{k, i}(x) \in L^{2}\left(B_{k}\right), i=1,2, \ldots, n$,

$$
\lim _{m \rightarrow \infty} \sum_{i=1}^{n} \int_{B_{k}}\left(\frac{\partial u_{m}}{\partial x_{i}}-v_{k, i}(x)\right)^{2} d x=0
$$

Let us define $\widetilde{v}_{k, i}(x)$ in the following way:

$$
\begin{aligned}
& \widetilde{v}_{k, i}(x)= \begin{cases}v_{k, i}(x) & \text { if } x \in B_{k}, \\
0 & \text { if } x \in B-B_{k},\end{cases} \\
& \widetilde{v}_{k, i}(x)=v_{k, i}(x) \quad \text { if } x \in B_{k},
\end{aligned}
$$

and

$$
\widetilde{v}_{k, i}(x)=0 \quad \text { if } x \in B-B_{k}
$$

Now define

$$
v_{i}(x)=\limsup _{k \rightarrow \infty} \widetilde{v}_{k, i}(x), \quad i=1,2, \ldots, n
$$

By definition it is clear that

$$
v_{i}(x)=v_{k, i}(x) \quad(\text { a.e. } d x) \text { on ball } B_{k}
$$

Thus the functions $v_{i}(x)$ are locally square integral on the ball $B$. Now we claim that $v_{i}(x)$, $i=1,2, \ldots, n$, is a Sobolev weak derivative of the function $u(x)$. To prove this, take

$$
\phi(x) \in C_{0}^{\infty}(B)
$$


The support

$$
\begin{aligned}
& \phi(x) \subseteq B_{k} \quad \text { for some } k \\
& \int_{B_{k}} \frac{\partial u_{m}}{\partial x_{i}} \phi(x) d x=-\int_{B_{k}} u_{m} \frac{\partial \phi_{m}}{\partial x_{i}} d x
\end{aligned}
$$

for any $m \geq m(k)$.

Hence passing to the limits as $m \rightarrow \infty$, we have

$$
\int_{B_{k}} v_{i}(x) \phi(x) d x=-\int_{B_{k}} u(x) \frac{\partial \phi}{\partial x_{i}} d x
$$

This shows $v_{i}(x), i=1,2, \ldots, n$, is a partial derivative of $u(x)$.

Again writing the inequality (2.6) for the ball $B_{k+1} \subseteq B$ and for the function $u_{1}(x)=0$, $u_{2}(x)=u_{m}(x)$, we get

$$
\int_{B_{k+l}}\left|\operatorname{grad} u_{m}(x)\right|^{2} h_{k+l}(x) d x \leq \frac{3}{2}\left\|u_{m}(x)\right\|^{2} \int_{B_{k+l}}\left|\Delta h_{k+l}(x)\right| d x
$$

Passing to the limit as $m \rightarrow \infty$

$$
\int_{B_{k+l}}|\operatorname{grad} u(x)|^{2} h_{k+l}(x) d x \leq \frac{3}{2}\|u(x)\|_{L^{\infty} B_{k+l}}^{2} \int_{B_{k+l}}\left|\Delta h_{k+l}(x)\right| d x .
$$

Since $B_{k}+1 \subseteq B$, taking the left hand integral on the small ball, we have

$$
\int_{B_{k}}|\operatorname{grad} u(x)|^{2} h_{k+l}(x) d x \leq \frac{3}{2}\|u(x)\|_{L^{\infty} B_{k+l}}^{2} \int_{B_{k+l}}\left|\Delta h_{k+l}(x)\right| d x .
$$

Now let the integer $l$ go to infinity; we have

$$
\int_{B_{k}}|\operatorname{grad} u(x)|^{2} h(x) d x \leq \frac{3}{2}\|u(x)\|_{L}^{\infty}(B) \int_{B}|\Delta h(x)| d x<\infty .
$$

If the left hand side is increasing and bounded, then it has a finite limit and so

$$
\int_{B}|\operatorname{grad} u(x)|^{2} h(x) d x<\frac{3}{2}\|u(x)\|_{L}^{\infty}(B) \int_{B}|\Delta h(x)| d x<\infty .
$$

Now we will give a proof of our main result.

Proof of Theorem 1.1 Take $u_{m, i}(x), i=1,2$, the mollification of the weak superharmonic functions $u_{i}(x), i=1,2$.

By the definition of mollification, we know that for a ball $B_{k+l}$, there exists an integer $m_{k+l}$ such that each function $u_{m, i}, i=1,2$, is a smooth superharmonic function on the ball $B_{k+l}$ if $m \geq m_{k+l}$.

Also we have the following convergence:

$$
\left\|u_{m, i}(x)-u_{i}(x)\right\| \rightarrow 0, \quad \text { if } m \rightarrow \infty, i=1,2 .
$$


Now we write the inequality (2.4) for the functions $u_{m, 1}(x)$ and $u_{m, 2}(x)$ for the ball $B_{k+l}$. We have

$$
\begin{aligned}
& \int_{B_{k+l}}\left|\operatorname{grad} u_{m, 2}(x)-\operatorname{grad} u_{m, 1}(x)\right|^{2} h_{k+l}(x) d x \\
& \leq \alpha_{k+l}\left[\frac{1}{2}\left\|u_{m, 2}-u_{m, 1}\right\|_{L_{\infty}\left(B_{k+l}\right)}^{2}+\left(\left\|u_{m, 2}-u_{m, 1}\right\|_{L_{\infty}\left(B_{k+l}\right)}\right)\right. \\
& \left.\quad \times\left(\left\|u_{m, 1}\right\|_{L_{\infty}\left(B_{k+l}\right)}+\left\|u_{m, 2}\right\|_{L_{\infty}\left(B_{k+l}\right)}\right)\right] .
\end{aligned}
$$

Passing to the limit as $m \rightarrow \infty$, we obtain

$$
\begin{aligned}
& \int_{B_{k+l}}\left|\operatorname{grad} u_{2}(x)-\operatorname{grad} u_{1}(x)\right|^{2} h_{k+l}(x) d x \\
& \leq \alpha_{k+l}\left[\frac{1}{2}\left\|u_{2}-u_{1}\right\|_{L_{\infty}\left(B_{k+l}\right)}^{2}+\left(\left\|u_{2}-u_{1}\right\|_{L_{\infty}\left(B_{k+l}\right)}\right)\right. \\
& \left.\quad \times\left(\left\|u_{1}\right\|_{L_{\infty}\left(B_{k+l}\right)}+\left\|u_{2}\right\|_{L_{\infty}\left(B_{k+l}\right)}\right)\right]
\end{aligned}
$$

Since the ball $B_{k} \subseteq B_{k+l}$, writing the left hand side for the smaller ball and passing to the limit $l \rightarrow \infty$, the above becomes

$$
\begin{aligned}
& \int_{B_{k}}\left|\operatorname{grad} u_{2}(x)-\operatorname{grad} u_{1}(x)\right|^{2} h_{\infty}(x) d x \\
& \quad \leq c_{\infty}\left[\frac{1}{2}\left\|u_{2}-u_{1}\right\|_{L_{\infty}(B)}^{2}+\left(\left\|u_{2}-u_{1}\right\|_{L_{\infty}(B)}\right)\left(\left\|u_{1}\right\|_{L_{\infty}(B)}+\left\|u_{2}\right\|_{L_{\infty}(B)}\right)\right] .
\end{aligned}
$$

By Theorem 3.1, we have

$$
\int_{B}\left|\operatorname{grad} u_{i}(x)\right|^{2} h(x) d x<\infty, \quad i=1,2
$$

Passing to the limit as $k \rightarrow \infty$, we obtain the required result.

For a continuous function in $\bar{B}$, Wilson and Zwick [4] described the best continuous subharmonic approximation. He found that the best subharmonic approximation of a continuous function $f(x)$ is just the greatest subharmonic minorant of the function. But in the case of a superharmonic approximation it will be smallest superharmonic majorant. The details are given below. In the problem when the analytic unknown exact solution must be superharmonic in the ball $B$, it is of interest to find numerical approximation $\epsilon_{0}$ that are superharmonic themselves. One expects that they will be better approximations to the unknown solution $u(x)$ than the ones somehow constructed through the uniform approximation $u_{h}(x)$. Suppose $u_{h}(x)$ is the uniform approximation to the unknown superharmonic function $u(x)$ in $\bar{B}$. Then $-u(x)$ will be the subharmonic function and $-u_{h}(x)$ will approximate $-u(x)$. We have

$$
\begin{aligned}
& -v_{h}(x)=\sup \left\{-g(x) \mid-g(x) \text { is subharmonic and }-g(x) \leq-u_{h}(x)\right\} \\
& -v_{h}(x)=\sup \left\{-g(x) \mid-g(x) \text { is subharmonic and } g(x) \geq u_{h}(x)\right\}
\end{aligned}
$$




$$
v_{h}(x)=\inf \left\{g(x) \mid g(x) \text { is superharmonic in } B \text { and } g(x) \geq u_{h}(x)\right\} \text {. }
$$

Denote

$$
\delta=\left\|u(x)-u_{h}(x)\right\|_{L^{\infty}}
$$

where

$$
\begin{aligned}
& \delta=\sup _{x \in B}\left|u-u_{h}\right|, \\
& \left|u-u_{h}\right| \leq \delta \quad \Rightarrow \quad-\delta \leq u-u_{h} \leq \delta .
\end{aligned}
$$

Thus

$$
\begin{aligned}
& \left.v_{h}(x) \geq u_{h}(x) \quad \text { (by the definition of } v_{h}(x)\right), \\
& v_{h}(x)+\delta \geq u_{h}(x)+\delta \geq u(x),
\end{aligned}
$$

so

$$
v_{h}(x)-u(x) \geq-\delta
$$

Also

$$
u(x)+\delta \text { is a majorant of } u_{h}(x)
$$

So

$$
\begin{aligned}
& u(x)+\delta \geq v_{h}(x) \\
& v_{h}(x)-u(x) \leq \delta .
\end{aligned}
$$

From (3.11) and (3.12)

$$
\left\|v_{h}(x)-u(x)\right\|_{L^{\infty}} \leq\left\|u_{h}(x)-u(x)\right\|_{L^{\infty}}
$$

Both $v_{h}(x)$ and $u(x)$ are superharmonic in $B$, and we also assume that they are continuous and bounded. By the use of inequality (1.4), we obtain the following important estimate:

$$
\begin{aligned}
& \int_{B\left(x_{0}, r\right)}\left|\operatorname{grad} v_{h}(x)-\operatorname{grad} u(x)\right|^{2} h(x) d x \\
& \quad \leq \frac{1}{2}\left\|u_{h}-u\right\|_{L_{\infty}\left(B\left(x_{0}, r\right)\right)}^{2}+\left(\left\|u_{h}-u\right\|_{L_{\infty}\left(B\left(x_{0}, r\right)\right)}\right)\left(\|u\|_{L_{\infty}\left(B\left(x_{0}, r\right)\right)}\right. \\
& \left.\quad+\left\|u_{h}\right\|_{L_{\infty}\left(B\left(x_{0}, r\right)\right)}\right) \int_{B\left(x_{0}, r\right)}|\Delta h(x)| d x .
\end{aligned}
$$


Authors' contributions

The first theorem for generalized domain and generalized weight function is given by JP. MSS and the M. Phil. student Mr. $\mathrm{AH}$ developed the convolution technique and also gave the existence of the Sobolov gradient. Mr. HUR developed the inequality for weak subsolutions. Mr. AMN developed the last inequality. All authors read and approved the final manuscript.

\section{Author details}

${ }^{1}$ Faculty of Textile Technology, University of Zagreb, Zagreb, 10000, Croatia. ${ }^{2}$ Department of Mathematics, University of Education, Lahore, Pakistan.

Received: 26 May 2015 Accepted: 29 November 2015 Published online: 15 December 2015

\section{References}

1. Shashiashvili, K, Shashiashvili, M: Estimation of the derivative of the convex function by means of its uniform approximation. JIPAM. J. Inequal. Pure Appl. Math. 6(4), Article 113 (2005)

2. Hussain, S, Shashiashvili, M: Discrete time hedging of the American option. Math. Finance 20(4), 647-670 (2010)

3. Hussain, S, Pečarić, J, Shashiashvili, M: The weighted square integral inequalities for the first derivative of the function of a real variable. J. Inequal. Appl. 2008, Article ID 343024 (2008)

4. Wilson, JM, Zwick, D: Best approximation by subharmonic functions. Proc. Am. Math. Soc. 114(4), 897-903 (1992)

\section{Submit your manuscript to a SpringerOpen ${ }^{\circ}$ journal and benefit from:}

- Convenient online submission

Rigorous peer review

- Immediate publication on acceptance

Open access: articles freely available online

High visibility within the field

- Retaining the copyright to your article 